\title{
Effects of fragrance compounds on growth of the silkworm Bombyx mori
}

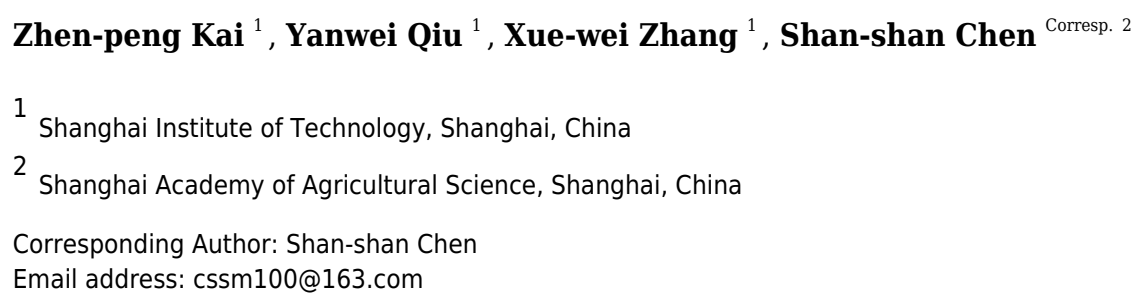

Due to the contamination and biological toxicity of some fragrance compounds, the environmental and ecological problems of such compounds have attracted more and more attention. However, studies of the toxicity of fragrance compounds for insects have been limited. The toxicity of 48 fragrance compounds for the silkworm Bombyx mori were investigated in this study. All of the fragrance compounds examined had no acute toxicity for B. mori larvae, but eight of them (menthol, maltol, musk xylene, musk tibeten, dibutyl sulfide, nerolidol, ethyl vanillin, and $\alpha$-amylcinnamaldehyde) exhibited chronic and lethal toxicity with $\mathrm{IC}_{50}$ values from 20 to $120 \mu \mathrm{M}$. In a long-term feeding study, musk tibeten, nerolidol, and musk xylene showed significant growth regulatory activity. They were also extremely harmful to the cocooning of $B$. mori, resulting in small, thin, and loose cocoons. Two important insect hormones, namely, juvenile hormone $(\mathrm{JH})$ and 20-hydroxyecdysone (20-E), were quantified in hemolymph following chronic exposure to musk tibeten, nerolidol, and musk xylene, respectively. Musk tibeten significantly increased JH titer and decreased the 20-E titer in hemolymph, and musk xylene had a significant inhibitory effect on JH titer and increased 20-E titer. Although nerolidol had no effect on hormone levels, exogenous $\mathrm{JH}$ mimic nerolidol increased the physiological effects of $\mathrm{JH}$ and significantly slowed the growth rate of $B$. mori larvae. The results showed that these fragrance compounds could interfere with the insect endocrine system, leading to death and abnormal growth. The risk to insects of residual fragrance compounds in the environment is worthy of attention. 
1

2

3

4

5

$6{ }^{1}$ School of Chemical and Environmental Engineering, Shanghai Institute of Technology,

7 Shanghai, 201418, China ${ }^{2}$ Institute of Agro-

8 food Standards and Testing Technologies, Shanghai Academy of Agricultural Science, Shanghai,

9201403 , China

10

11 Corresponding Author:

12 Shan-shan Chen ${ }^{2}$

13 No. 1000 Jinqi Road, Shanghai, 201403, China

14 Email address: shanshanchen2013@saas.sh.cn 
16 Abstract: Due to the contamination and biological toxicity of some fragrance compounds, the

17 environmental and ecological problems of such compounds have attracted more and more

18 attention. However, studies of the toxicity of fragrance compounds for insects have been limited.

19 The toxicity of 48 fragrance compounds for the silkworm Bombyx mori were investigated in this

20 study. All of the fragrance compounds examined had no acute toxicity for B. mori larvae, but

21 eight of them (menthol, maltol, musk xylene, musk tibeten, dibutyl sulfide, nerolidol, ethyl

22 vanillin, and $\alpha$-amylcinnamaldehyde) exhibited chronic and lethal toxicity with $\mathrm{IC}_{50}$ values from

2320 to $120 \mu \mathrm{M}$. In a long-term feeding study, musk tibeten, nerolidol, and musk xylene showed

24 significant growth regulatory activity. They were also extremely harmful to the cocooning of $B$.

25 mori, resulting in small, thin, and loose cocoons. Two important insect hormones, namely,

26 juvenile hormone ( $\mathrm{JH})$ and 20-hydroxyecdysone (20-E), were quantified in hemolymph

27 following chronic exposure to musk tibeten, nerolidol, and musk xylene, respectively. Musk tibeten significantly increased $\mathrm{JH}$ titer and decreased the 20-E titer in hemolymph, and musk

29 xylene had a significant inhibitory effect on JH titer and increased 20-E titer. Although nerolidol had no effect on hormone levels, exogenous JH mimic nerolidol increased the physiological effects of $\mathrm{JH}$ and significantly slowed the growth rate of $B$. mori larvae. The results showed that these fragrance compounds could interfere with the insect endocrine system, leading to death and abnormal growth. The risk to insects of residual fragrance compounds in the environment is worthy of attention.

KEYWORDS: toxicity; fragrance compounds; Bombyx mori; juvenile hormone; 20- 
37 hydroxyecdysone 
Fragrance compounds are a group of structurally diverse additives used in a wide variety of

consumer products. In recent years, there have been increasing reports of pollution of fragrance

compounds in the environment and detection of them in organisms (Weinberg et al., 2011;

Vecchiato et al., 2018; Lou et al., 2016). Tasselli et al (2021) showed the current technologies

are not enough efficient in removing the polycyclic musk fragrances from wastewaters in Italy.

43 The preliminary ecological risk assessment showed that synthetic musk fragrances also pose high risk to aquatic organisms in the riverine and estuarine environment in Thailand (Juksu et al., 2020). HHCB-lactone was also found with the highest concentration up to $79501 \mathrm{ng} / \mathrm{g} \mathrm{(dw)} \mathrm{in}$ the sludge. Low removal efficiency range from $-37 \%$ (HHCB-lactone) to $58 \%$ (AHTN) were found for four musks. In response to the contamination and biological toxicity of some fragrance compounds (such as synthetic musk fragrances), a number of studies have focused on environmental and ecological problems related to them. Synthetic musk fragrances have been detected in mussels (Parolini et al., 2015), fishes (Blahova et al., 2018), birds (Kannan et al., 2005), marine mammals, and sharks (Nakata et al., 2007; Nakata 2005), as well as in human tissues and milk (Schiavone et al., 2010; Moon et al., 2012; Kang et al., 2010). Polycyclic musks

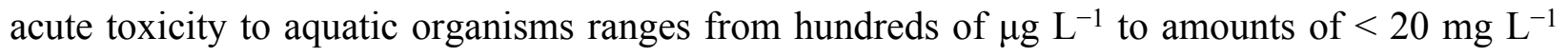

54 (Tumová et al., 2019). Schreurs et al. (2004) reported that AHTN and HHCB also have antiestrogenic effects on the human estrogen receptor in vitro. Multiple studies have shown that synthetic musk can increase cancer risk (Zhang et al., 2017). Musk xylene exhibits a genotoxic activity even after short exposure times following a DNA damage assay (Rocco et al., 2015). 
59 for the stable maintenance of the balance of the ecosystem (Potts et al., 2016). The silkworm is

60 the main raw material source of silk and thus plays an important role in human economic life and

61 cultural history. The silkworm is also widely used in entomological research as a model

62

63

64

organism. Some fragrances and flavors, especially certain essential plant oils have long been reputed to repel pests (Isman, 2000). Despite the importance of silkworm and other insects, there have been few studies of the effects of daily chemical fragrance compounds on insects. In this study, the acute and chronic toxicity of 46 commonly used commercial fragrance compounds (including natural and synthetic compounds) were performed tested to determine their lethal toxicity and regulatory activity on the growth of Bombyx mori. The effects of the fragrance compounds on cocooning were also determined in a long-term feeding study. Finally, juvenile hormone (JH) and 20-hydroxyecdysone (20-E) were quantified in silkworm hemolymph to test the possible causes of toxicity.

\section{MATERIALS AND METHODS}

\section{Insect}

Larvae of the silkworm Bombyx mori strain p50 (DaZao) were raised from eggs provided by the Institute of Sericulture and System Biology at the Southwest University of China. The larvae were reared on the fresh mulberry leaves at $26 \pm 1^{\circ} \mathrm{C}, 70 \pm 5 \%$ humidity with a photoperiod of $12 \mathrm{~h}$ light and $12 \mathrm{~h}$ dark.

\section{Chemicals}

All fragrance compounds (Table S1) were purchased from TCI Shanghai, China. JH I, JH II, JH III and 20-E were purchased from Toronto Research Chemicals (Toronto, ON, Canada) and 
80

81

82

83

Sigma-Aldrich (St. Louis, MO), respectively. The internal standards JH III-D3 and ponasterone A were purchased from J\&K Scientific Ltd. (Beijing, China).

\section{Assays for to determine the impact of feeding on B. mori larval growth and mortality}

Healthy and active larvae of similar size (larval length $3 \pm 0.5 \mathrm{~mm}$ ) at the end of the first ecdysis were randomly selected and placed in plastic containers. In the acute feeding study, newly molted healthy larvae at the second stadium were randomly selected and fed with a small piece mulberry leaves contaminated with $2 \mu \mathrm{L}$ fragrance solution at concentration $1 \mathrm{mM}$, and then reared on normal fresh mulberry leaves. Larvae were observed after $24 \mathrm{~h}$, when dead larvae were recorded and removed. In the long-term feeding study, the larvae were exposed to the fragrance compounds from the second instar $(2 \mu \mathrm{L}$ for insects at the second instar, $4 \mu \mathrm{L}$ at the third instar, and $6 \mu \mathrm{L}$ at the fourth and fifth instars), administered in the same way as in the acute feeding study. Each animal was treated with 2-6 pmol day ${ }^{-1}$ (approximately 1 ng day ${ }^{-1}$ ) fragrance compounds. Each day, after the larvae had finished ingesting the contaminated mulberry leaves, fresh and clean leaves were fed to maintain their dietary requirements. The fragrance compounds were dissolved in dimethyl sulfoxide and then mixed with pure water. The concentrations ranged from 0.001 to $10 \mathrm{mM}$. The concentrations tested for each compound were shown in Table S1.

Larval mortality and insect wet weight were recorded every day after treatment (Wang et al., 1999). Controls were treated with the solvent. There have three replications (done on separate days) at each dose and each replication contained 5-7 larvae.

\section{Quantitative assays of JH in hemolymph using LC-MS/MS}

Newly molted fifth-instar B. mori (day 0) were used in this study. The hemolymph was 
101

102

103

104

105

106

107

108

109

110

111

112

113

114

115

116

117

118

119

120

121

collected in a microsyringe after cutting an abdominal leg. A volume of $50 \mu \mathrm{L}$ hemolymph was immediately transferred to a $500 \mu \mathrm{L}$ glass centrifuge tube containing $50 \mu \mathrm{L}$ acetonitrile, $50 \mu \mathrm{L}$ sodium chloride $\left(0.9 \%\right.$, wt $\left.\mathrm{v}^{-1}\right)$ solution, and $5 \mathrm{ng}$ JH III-D3 as an internal standard. The sample was extracted twice with $100 \mu \mathrm{L}$ hexane following vigorous vortexing and centrifuged at $2500 \times$ g for 5 min (Kai et al., 2018). The hexane phase (upper layer) was removed and transferred to a new glass vial, and then dried under nitrogen flow. The residue was dissolved in $1 \mathrm{~mL}$ acetonitrile. The measurement of JHs was determined using a liquid chromatography method coupled to electrospray tandem mass spectrometry (LC-MS/MS) reported by Ramirez et al. (2020).

\section{Quantitative assays of 20-E in whole body using UPLC-MS/MS}

The hemolymph of B. mori larvae (4 days into the fourth instar) was collected as described above, and $50 \mu \mathrm{L}$ hemolymph was immediately transferred into $500 \mu \mathrm{L}$ methanol. Ponasterone A (10 $\mathrm{ng} \mathrm{mL}^{-1}$ ) was used as the internal standard in this study. The mixture was vortexed vigorously and centrifuged at $2500 \times \mathrm{g}$ for $10 \mathrm{~min}$. The supernatant was collected in a new microtube, and the pellet was re-extracted with $500 \mu \mathrm{L}$ methanol. The combined methanol extracts were evaporated under a stream of nitrogen. The residue was dissolved in $100 \mu \mathrm{L} 80 \%$ methanol in water (Furuta et al., 2010). The quantitative assay for 20-E was determined using a UPLC-MS/MS system, consisting of Waters ACQUITY UPLC I-Class (Waters Corp., Milford, MA), coupled with AB SCIEX TRIPLE QUAD ${ }^{\mathrm{TM}} 5500$ mass spectrometer from AB Sciex (Framingham, MA, USA).

An ACQUITY UPLC®BEH C18 column $(2.1 \mathrm{~mm} \times 100 \mathrm{~mm}, 1.7 \mu \mathrm{m})$ from Waters was 
122

123

124

125

126

127

128

129

130

131

132

133

134

135

136

137

138

139

140

141

142

used for separation at $40^{\circ} \mathrm{C}$ with a sample injection volume of $3 \mu \mathrm{L}$. A binary mobile phase was composed of (A) methanol and (B) $0.1 \%$ formic acid in water. A linear mobile phase gradient started at 10\% A (0-1 min) and went to $90 \% \mathrm{~A}$ at $4 \mathrm{~min}$ (held until $7 \mathrm{~min}$ ), followed by a gradient to $10 \% \mathrm{~A}$ at $7.1 \mathrm{~min}$ (held until $8 \mathrm{~min}$ ) at a flow rate of $0.3 \mathrm{~mL} \mathrm{~min}{ }^{-1}$. The MS determination was performed in positive electrospray mode with monitoring of the two most abundant MS/MS (precursor/product) ion transitions using scheduled multiple-reaction monitoring (MRM) program for each analyte. The ion source conditions were as follows: ion spray voltage, $5.5 \mathrm{kV}$; block source temperature, $500^{\circ} \mathrm{C}$; ion source gas 1 pressure, $50 \mathrm{psi}$; ion source gas 2 pressure, $50 \mathrm{psi}$; curtain gas pressure, $38 \mathrm{psi}$; and collision gas (argon) pressure, 8 psi. Data acquisition and processing were carried out by Analyst software version 1.6.1 (Applied Biosystems, Foster City, CA, USA). The retention times for 20-E and ponasterone A were 3.86 $\min$ and $4.43 \mathrm{~min}$, respectively. The quantification transition for 20 -E was $481.3 \rightarrow 445.3$ (declustering potential [DP]: $132 \mathrm{~V}$, CE: $24 \mathrm{~V}$ ). The confirmation transitions were $481.3 \rightarrow 371.2$ (DP: $159 \mathrm{~V}, \mathrm{CE}: 23 \mathrm{~V}$ ) and $481.3 \rightarrow 427.5$ (DP: $138 \mathrm{~V}, \mathrm{CE}: 23 \mathrm{~V}$ ), respectively. For the internal standard ponasterone A, the quantification transition was $465.5 \rightarrow 429.3$ (DP: $150 \mathrm{~V}$, CE: $23 \mathrm{~V}$ ), and the confirmation transitions were $465.5 \rightarrow 295.3$ (DP: $150 \mathrm{~V}, \mathrm{CE}: 30 \mathrm{~V}$ ) and $465.5 \rightarrow 411.2$ (DP: $160 \mathrm{~V}, \mathrm{CE}: 26 \mathrm{~V})$.

\section{Statistics}

Comparisons of the effects of fragrance compounds on larval stadium and the growth of larva were analyzed with a pooled $t$-test. The threshold for statistical significance was set at $95 \%$ $(\mathrm{P}=0.05)$. Data were presented as percentages and were log-transformed before statistical 
143 analyses. Dose-response curves were prepared using the software GraphPad Prism version 5.0.

144 Values are expressed as means \pm standard errors (SEM), with $\mathrm{N}$ indicating the number of

145 samples measured $(\mathrm{N}=15-21)$.

\section{RESULTS}

\section{Larval mortality following oral administration}

148 A stomach toxicity test was performed to determine the larvicidal effects of the fragrance compounds in this study. After $24 \mathrm{~h}$ exposure following feeding at concentration $1 \mathrm{mM}$, no larvae died. This suggests that the fragrance compounds in this study have no acute toxicity for $B$. mori larvae. However, eight fragrance compounds (menthol, maltol, musk xylene, musk tibeten, dibutyl sulfide, nerolidol, ethyl vanillin, and $\alpha$-amylcinnamaldehyde) showed chronic toxicity. Most larvae that commenced feeding with these eight compounds from the second stadium died prior to pupation. The $\mathrm{LC}_{50}$ value and mortality at $0.1 \mathrm{mM}$ of each compound are shown in Table

1. These results show that the eight fragrance compounds have chronic and lethal toxicity for $B$. mori larvae. In the dead larvae, the most striking characteristic was the darkening of the cuticle in some animals and molting disturbances. This phenomenon suggested that these fragrance compounds might interfere with insect hormone levels, leading to death (Monger et al., 1982).

No antifeedant effect was found in these experiments.

160

161

\section{Growth regulation}

A long-term feeding study with fragrance compounds at $1 \mu \mathrm{M}$ was performed to identify

162 their effects on growth. We fed larvae with fragrance compounds starting at the second instar 
163 (Day 0), and recorded the number of days from hatching to pupation (larvae that died before 164 pupation were not recorded). Figure 1 shows musk tibeten and nerolidol significantly slowed the

165

166

167 168 169 growth rate of $B$. mori larvae. However, musk xylene caused precocious pupation (Figure 1). For musk tibeten, nerolidol and musk xylene, the times from newly hatched larvae to pupation were 31.6, 29.9, and 24.4 days, respectively. In the control group (not fed inhibitors), the interval was 27.4 days. Figure 2 clearly shows that larvae fed with these three fragrance compounds were significantly smaller than the control group. Four larval groups were hatched on the same day (Day 0, second instar); one was fed normal food as control, and the others were treated with 1 $\mu \mathrm{M}$ musk tibeten, nerolidol, and musk xylene, respectively. The difference in wet weight was readily apparent by comparison with the control insects at the fifth instar.

The effects of musk tibeten, nerolidol, and musk xylene on cocooning of $B$. mori were also determined with a long-term feeding study at $1 \mu \mathrm{M}$ as above. The mean wet weight of cocoon in treatment was significantly less than in the controls (Figure 3). Compared to the control, the wet weight of cocoon exposed to musk tibeten, nerolidol, and musk xylene lost $0.34 \mathrm{~g} \mathrm{cocoon}^{-1}, 0.23$ $\mathrm{g} \mathrm{cocoon}^{-1}$, and $0.40 \mathrm{~g} \mathrm{cocoon}^{-1}$, respectively. There were no significant differences between treatments and the controls on rate of cocooning.

Although mortality in the low-dose chronic exposure to fragrance compounds was minimal, the growth of $B$. mori larvae was greatly interfered with, and the mean wet weight of larvae in each larval instar were also reduced following chronic exposure to musk tibeten, nerolidol, and musk xylene. These three fragrance compounds were also extremely harmful to the cocooning of B. mori. The affected larvae exhibited behavioral responses, such as obstruction of cocoon- 
184 185

making or spinning, feeble pulse rate and final death of surviving larvae. Some larvae made only thin and loose cocoons.

\section{Effects on JH and 20-E titer in hemolymph by treatment with fragrance compounds}

Hormones are generated and used by insects to regulate physiological, developmental, and behavioral events. Juvenile hormones and ecdysteroids are two important categories of insect hormone. Ecdysteroids are a family of steroid hormones. Their most common form in insects is 20-E, which is converted from ecdysone in the fat body or epidermis (Horn et al., 1966). To study the possible causes of chronic toxicity, JHs (JH I, JH II and JH III) and 20-E were quantified in hemolymph following chronic exposure to musk tibeten, nerolidol, and musk xylene. Larvae were fed fragrance compounds (an approximate exposure of 1 ng day ${ }^{-1}$ ), as described in Section 3.2. Figure 4 shows that musk tibeten and musk xylene had significant effects on JHs titer following treatment in vivo, while nerolidol was inactive. It is interesting that musk tibeten significantly increased JHs titer in hemolymph, whereas musk xylene had significant inhibitory effect. In this study, other synthetic musk fragrances (such as musk ketone, musk AHMT, and phantolide) did not demonstrate any growth-regulatory effects on B. mori.

The 20-E titer in the hemolymph of B. mori larvae (day 4 of the fourth instar) was determined by UPLC-MS/MS (Figure 5). Treatment with musk tibeten significantly decreased the 20-E titer in hemolymph, and musk xylene had a significantly increased effect. This result was contrary to the JH titer. Musk tibeten increased JH titer but reduced 20-E titer in hemolymph, which resulted in the retardation of the growth of $B$. mori larvae. Must xylene had a significant inhibitory effect on JH titer but increased the 20-E titer in hemolymph. This caused precocious 
205

206

207

208

209

210

211

212

213

214

215

216

217

218

219

220

221

222

223

224

225

pupation. Nerolidol also had no effect on 20-E titer. The quantitative assays for JHs and 20-E titer indicate possible causes of growth regulation by these three fragrance compounds.

\section{DISCUSSION}

Geraniol and geranylgeraniol have been shown to inhibit JH biosynthesis by the corpora allata in lepidopteran and blattarian insects in vitro (Kai et al., 2018; Sperry and Sen, 2001).

Coumarin has been demonstrated as an ovicide inhibitor against fruit fly Drosophila

melanogaster (Shuhei and Kazuyoshi, 1980). Toxicity tests illustrated the fumigant toxicity of

1,8-cineole against stored-grain insects (Lee et al., 2004). However, these four fragrance

compounds above had no lethal toxicity to $B$. mori larvae following the stomach toxicity test in

this study. This suggests that different species of insects have different sensitivities to given

fragrance compounds.

Our result suggests that the structural differences in fragrances have a great influence on

toxicity. Musk tibeten and musk xylene are aromatic nitro compounds, and musk ketone has an

aldehyde group in addition to the nitro group, however musk AHMT and phantolide do not

contain nitro groups. Nerolidol is a mimic of $\mathrm{JH}$, which has a similar function, but it has no

effect on JH biosynthesis and metabolism (Burdette, 1964). In hemolymph, exogenous JH mimic

nerolidol increases the physiological effects of $\mathrm{JH}$ and significantly slows the growth of $B$. mori

larvae.

In previous studies, some fragrance compounds have been demonstrated to have contact,

fumigant, antifeedant, attractant, and repellent insecticidal actions against specific pests (Isman, 2000). Due to the extensive use of fragrance compounds and the growth of the fragrance industry, 
226 fragrance residues can be detected in the environment. Concentrations of fragrance compounds

227 in wastewater were found in concentration at $\mu \mathrm{g} \mathrm{L}^{-1}$ in a study in Italy (Tasselli et al., 2021). In

228 the long-term feeding study with fragrance compounds at $1 \mu \mathrm{M}$ was performed to identify their

229 effects on the growth of insect. Residual fragrance compounds may cause damage to the

230 sericulture industry and may be harmful to other insects in the environment. The current leakage

231 concentration may not immediately affect the ecology of insects. However, there are concerns

232 about the deterioration of environmental pollution in the future.

233 CONCLUSIONS

234 Although the fragrance compounds in this study have no acute toxicity for $B$. mori larvae,

235 some exhibit chronic and lethal toxicity. In a long-term feeding study, musk tibeten, nerolidol,

236 and musk xylene have significant growth-regulatory activity with an approximate exposure of 1

$237 \mathrm{ng} \mathrm{day}^{-1}$. They were also extremely harmful to the cocooning of B. mori. Larval exposure to

238 these fragrance compounds produced small, thin, and loose cocoons. The quantitative assays for

$239 \mathrm{JH}$ and 20-E titer in hemolymph showed that these fragrance compounds could interfere with

240 hormone levels in insects, leading to death and abnormal growth. The risk to insects of residual

241 fragrance compounds in the environment is worthy of attention.

243 Acknowledgments

244 This work was supported by grant from the National Natural Science Foundation of China (No.

245 21877126).

References 
247

248

249

250

251

252

253

254

255

256

257

258

259

260

261

262

263

264

265

266

267

Blahova J, Divisova L, Plhalova L, Enevova V, HostovskyM, Doubkova V, Marsalek P,

Fictum P, Svobodova Z. 2018. Multibiomarker responses of juvenile stages of zebrafish

(Danio rerio) to subchronic exposure to polycyclic musk tonalide. Arch Environ Contam Toxicol 74:568-576. DOI 10.1007/s00244-017-0484-8.

Burdette WJ. 1964. The significance of invertebrate hormones in relation to differentiation. Cancer Res 24: 521-536 DOI 10.1090/S0002-9904-1909-01740-9.

Furuta K, Fujita N, Ibushi T, Shiotsuki T, Yamada N, Kuwano E. 2010. Synthesis and antijuvenile hormone activity of ethyl 4-[(6-substituted 2,2-dimethyl-2h-chromen-7yl)methoxy]benzoates. J. Pestic. Sci 35: 405-411 DOI 10.1584/jpestics.G09-71.

Furuta K, Ichikawa A, Murata M, Kuwano E, Shiotsuki T. 2013. Determination by LC-MS of juvenile hormone titers in hemolymph of the silkworm, Bombyx mori. Biosci. Biotech. Bioch 77: 988-991 DOI 10.1271/bbb.120883.

Horn DHS, Middleton EJ, Wunderlich JA, Hampshire F. 1966. Identity of the moulting hormones of insects and crustaceans. Chem. Commun 142: 339-340 DOI 10.1039/c19660000339.

Isman MB. 2000. Plant essential oils for pest and disease management. Crop Prot 19: 603-608 DOI 10.1016/S0261-2194(00)00079-X.

Juksu K, Liu YS, Zhao JL, Yao L, Sarin C, Sreesai S, Klomjek P, Traitangwong A, Ying GG. 2020. Emerging contaminants in aquatic environments and coastal waters affected by urban wastewater discharge in Thailand: An ecological risk perspective. Ecotox Environ Safe 204: 110952. DOI 10.1016/j.ecoenv.2020.110952. 
268

269

270

271

272

273

274

275

276

277

278

279

280

281

282

283

284

285

286

287

288

Kai ZP, Yin Y, Zhang ZR, Huang J, Tobe SS, Chen SS. 2018. A rapid quantitative assay for juvenile hormones and intermidiates in the biosynthetic pathway using gas chromatography tandem mass spectrometry. J. Chromatogr. A 1538: $\quad$ 67-74 DOI 10.1016/j.chroma.2018.01.030.

Kang CS, Lee JH, Kim SK, Lee KT, Lee JS, Park PS, Yun SH, Kannan K., Yoo YW, Ha JY, Lee SW. 2010. Polybrominated diphenyl ethers and synthetic musks in umbilical cord serum, maternal serum, and breast milk from Seoul, South Korea. Chemosphere 80: 116122 DOI 10.1016/j.chemosphere.2010.04.009.

Kannan K, Reiner JL, Yun SH, Perrotta EE, Tao L, Johnson-Restrepo B, Rodan BD. 2005. Polycyclic musk compounds in higher trophic level aquatic organisms and humans from the United States. Chemosphere 61: 693-700 DOI 10.1016/j.chemosphere.2005.03.041.

Lee BH, Annis PC, Tumaalii F, Choi WS. 2004. Fumigant toxicity of essential oils from the myrtaceae family and 1,8-cineole against 3 major stored-grain insects. J. Stored Prod. Res 40: 553-564 DOI 10.1016/j.jspr.2003.09.001.

Lou Y, Wang J, Wang L, Shi L, Yu Y, Zhang M. 2016. Determination of synthetic musks in sediments of Yellow River delta wetland. China Bull Environ Contam Toxicol 97: 78-83. DOI 10.1007/s00128-016-1814-7.

Monger DJ, Lim WA, Kézdy FJ, Law, JH. 1982. Compactin inhibits insect hmg-coa reductase and juvenile hormone biosynthesis. Biochem. Bioph. Res. Co 105: 1374-1380 DOI 10.1016/0006-291x(82)90939-1.

Moon HB, Lee DH, Lee YS, Kannan K. 2012. Occurrence and accumulation patterns of 
289

290

291

292

293

294

295

296

297

298

299

300

301

302

303

304

305

306

307

308

309

polycyclic aromatic hydrocarbons and synthetic musk compounds in adipose tissues of Korean females. Chemosphere 86: 485-490 DOI 10.1016/j.chemosphere.2011.10.008.

Nakata H. 2005. Occurrence of synthetic musk fragrances in marine mammals and sharks from Japanese coastal waters. Environ. Sci. Technol 39: 3430-3434 DOI 10.1021/es0501991.

Nakata H, Sasaki, H, Takemura A, Yoshioka M, Tanabe S, Kannan K. 2007. Bioaccumulation, temporal trend, and geographical distribution of synthetic musks in the marine environment. Environ. Sci. Technol 41: 2216-2222 DOI 10.1021/es0623818.

Parolini M, Magni S, Traversi I, Villa S, Finizio A, Binelli A. 2015. Environmentally relevant concentrations of galaxolide (HHCB) and tonalide (AHTN) induced oxidative and genetic damage in Dreissena polymorpha. $J$ Hazard Mater 285:1-10. DOI 10.1016/j.jhazmat.2014.11.037.

Potts SG, Imperatriz-Fonseca V, Ngo HT, Aizen MA, Biesmeijer JC, Breeze TD, Dicks LV, Garibaldi LA, Hill R, Settele J, Vanbergen AJ. 2016. Safeguarding pollinators and their values to human well-being. Nature 540, 220-229 DOI 10.1038/nature20588.

Ramirez CE, Nouzova M, Michalkova V, Fernandez-Lima F, Noriega FG. 2020. Common structural features facilitate the simultaneous identification and quantification of the five most common juvenile hormones by liquid chromatography-tandem mass spectrometry. Insect Biochem Molec 116: 103287 DOI 10.1016/j.ibmb.2019.103287.

Rocco L, Mottola F, Santonastaso M, Saputo V, Cusano E, Costagliola D, Suero T, Pacifico S, Stingo V. 2015. Anti-genotoxic ability of $\alpha$-tocopherol and Anthocyanin to counteract fish DNA damage induced by musk xylene. Ecotoxicology 24: 2026-2035 DOI 
311

312

313

314

315

316

317

318

319

320

321

322

323

324

325

326

327

328

329

330

Schiavone A, Kannan K, Horii Y, Focardi S, Corsolini S. 2010. Polybrominated diphenyl ethers, polychlorinated naphthalenes and polycyclic musks in human fat from Italy: comparison to polychlorinated biphenyls and organochlorine pesticides. Environ. Pollut 158: 599-606 DOI 10.1016/j.envpol.2009.08.011.

Schreurs RHMM, Sonneveld E, Jansen JHJ, Seinen W, Van der Burg B. 2004. Interaction of polycyclic musks and UV filters with the estrogen receptor (ER), androgen receptor (AR), and progesterone receptor (PR) in reporter gene bioassays. Toxicol. Sci 83: 264-272 DOI 10.1093/toxsci/kfi035.

Shuhei N, Kazuyoshi K. 1980. Coumarin and euponin, two inhibitors for insect development from leaves of Eupatorium japonicum. Agric. Biol. Chem 44: 2893-2899 DOI 10.1080/00021369.1980.10864436.

Sperry AE, Sen SE. 2001. Farnesol oxidation in insects: evidence that the biosynthesis of insect juvenile hormone is mediated by a specific alcohol oxidase. Insect Biochem. Mol. Bio 31: 171-178 DOI 10.1016/s0965-1748(00)00115-6.

Tasselli S, Valenti E, Guzzella L. 2021. Polycyclic musk fragrance (PMF) removal, adsorption and biodegradation in a conventional activated sludge wastewater treatment plant in Northern Italy. Environ Sci Pollut $R$ In press. DOI 10.1007/s11356-021-13433-4.

Tumová J, Šauer P, Golovko O, Ucun OK, Grabic R, Máchová J, Kroupová HK. 2019. Effect of polycyclic musk compounds on aquatic organisms: a critical literature review supplemented by own data. Sci Total Environ 651:2235-2246. DOI 
332

333

334

335

336

337

338

339

340

341

342

343

344

Vecchiato M, Turetta C, Patti B, Barbante C, Piazza R, Bonato T, Gambaro A. 2018. Distribution of fragrances and PAHs in the surface seawater of the sicily channel, central mediterranean. Sci. Total Environ 634: 983-989 DOI 10.1016/j.scitotenv.2018.04.080.

Wang J, Yin DQ, Lu GF, Zhou FF. 1999. Effects of dimehypo (disodium 2methylaminotrimethylene di thiosulfonate) on growth and cocooning of the silkworm, Bombyx mori (lepidoptera: saturnidae). Pest Manag. Sci 55: 1070-1076. DOI 10.1002/(SICI)1096-9063(199911)55:11<1070::AID-PS51>3.0.CO;2-P.

Weinberg I, Dreyer A, Ebinghaus R. 2011. Landfills as sources of polyfluorinated compounds, polybrominated diphenyl ethers and musk fragrances to ambient air. Atmos Environ 45: 935-941. DOI 10.1016/j.atmosenv.2010.11.011.

Zhang YY, Huang LX, Zhao YJ, Hu TH. 2017. Musk xylene induces malignant transformation of human liver cell line L02 via repressing the TGF- $\beta$ signaling pathway. Chemosphere 168: 1506-1514 DOI 10.1016/j.chemosphere.2016.12.001. 
347 Figure 1. Number of days from hatching to pupation following a long-term feeding study with 348 musk tibeten, nerolidol, and musk xylene at $1 \mu \mathrm{M}$. B. mori larvae were fed with fragrance 349 compounds starting with the second instar, and the number of days from hatching to pupation was recorded. Values represent means \pm SEMs, $* * * * p<0.0001$.

Figure 2. Effects on larval weights of $B$. mori at the fifth instar in the chronic toxicity assay.

Four larval groups hatched on the same day (Day 0, second instar); one was fed normal food as control, and the others were treated with $1 \mu \mathrm{M}$ musk tibeten, nerolidol, and musk xylene, respectively. Each point represents mean $\pm \mathrm{SD} . N=20-21$ individuals.

Figure 3. Effects of musk tibeten, nerolidol, and musk xylene on the mean wet weight of cocoon in the chronic toxicity assay. Values represent means $\pm \mathrm{SEM}, * * * * p<0.0001$.

Figure 4. Effects of musk tibeten, nerolidol, and musk xylene on hemolymph JHs titer of newly molted fifth instar B. mori (day 0). Values represent means \pm SEM, $* * * * p<0.0001$.

Figure 5. Effects of musk tibeten, nerolidol, and musk xylene on hemolymph 20-E titer of $B$. 


\section{Table $\mathbf{1}$ (on next page)}

The $\mathrm{LC}_{50}$ values of fragrance compounds in the chronic toxicity assay

The $\mathrm{LC}_{50}$ values of fragrance compounds in the chronic toxicity assay 
1 Table 1. The $\mathrm{LC}_{50}$ values of fragrance compounds in the chronic toxicity assay

\begin{tabular}{lcc}
\hline Compound & $\begin{array}{c}\text { LC }_{50} \text { value } \\
(\mu \mathrm{M})(95 \% \mathrm{CI})\left(\text { nonlinear fit model, } \mathrm{df} ; R^{2}\right)\end{array}$ & Mortality at $0.1 \mathrm{mM}(\%)$ \\
\hline Menthol & $76.6(46.6-125.9)\left(\mathrm{df}=25 ; R^{2}=0.95\right)$ & $67 \pm 3$ \\
Maltol & $56.3(39.2-80.8)\left(\mathrm{df}=29 ; R^{2}=0.96\right)$ & $67 \pm 3$ \\
Musk xylene & $60.7(29.3-125.6)\left(\mathrm{df}=21 ; R^{2}=0.92\right)$ & $67 \pm 4$ \\
Musk tibeten & $88.1(52.3-148.4)\left(\mathrm{df}=27 ; R^{2}=0.97\right)$ & $65 \pm 5$ \\
Dibutyl sulfide & $118.1(66.2-210.7)\left(\mathrm{df}=27 ; R^{2}=0.90\right)$ & $51 \pm 5$ \\
Nerolidol & $63.2(23.5-170.0)\left(\mathrm{df}=25 ; R^{2}=0.85\right)$ & $66 \pm 15$ \\
Ethyl vanillin & $23.8(10.8-52.4)\left(\mathrm{df}=23 ; R^{2}=0.95\right)$ & $100 \pm 0$ \\
$\alpha$-amylcinnamaldehyde & $96.2(46.5-199.0)\left(\mathrm{df}=25 ; R^{2}=0.93\right)$ & $55 \pm 8$ \\
\hline
\end{tabular}

2 The repeat number of each concentration was $15-21 ; \mathrm{CI}=$ confidence intervals; $\mathrm{df}=$ degrees of

3 freedom.

4 
Figure 1

Number of days from hatching to pupation following a long-term feeding study with musk tibeten, nerolidol, and musk xylene at $1 \mu \mathrm{M}$.

B. mori larvae were fed with fragrance compounds starting with the second instar, and the number of days from hatching to pupation was recorded. Values represent means \pm SEMs, $* * * * p<0.0001$.

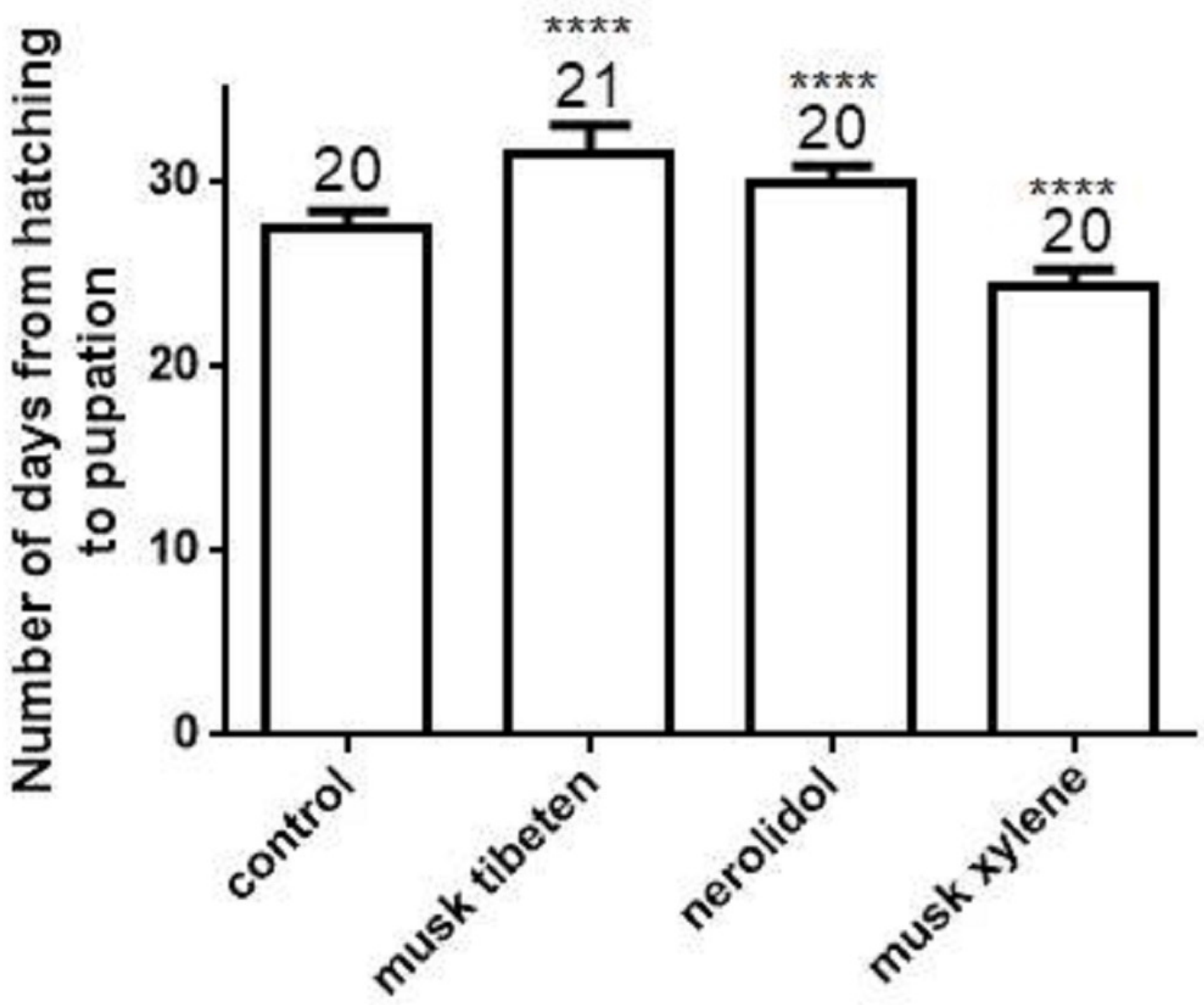


Figure 2

Effects on larval weights of $B$. mori at the fifth instar in the chronic toxicity assay.

Four larval groups hatched on the same day (Day 0, second instar); one was fed normal food as control, and the others were treated with $1 \mu \mathrm{M}$ musk tibeten, nerolidol, and musk xylene, respectively. Each point represents mean $\pm \mathrm{SD}$. $N=20-21$ individuals.

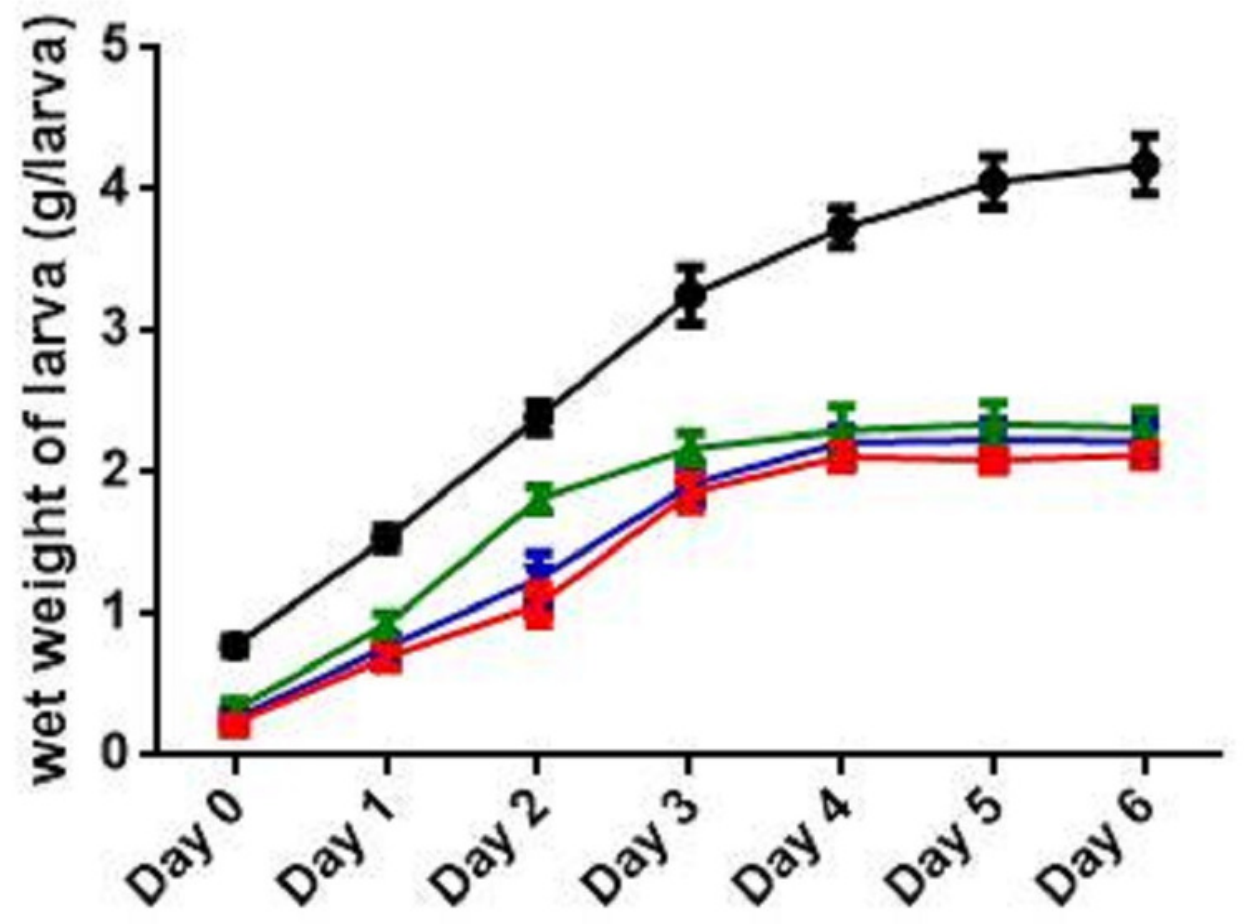

$\rightarrow$ control

-2- musk tibeten

- nerolidol

* musk xylene 
Figure 3

Effects of musk tibeten, nerolidol, and musk xylene on the mean wet weight of cocoon in the chronic toxicity assay.

Values represent means \pm SEM, ${ }^{* * * *} p<0.0001$

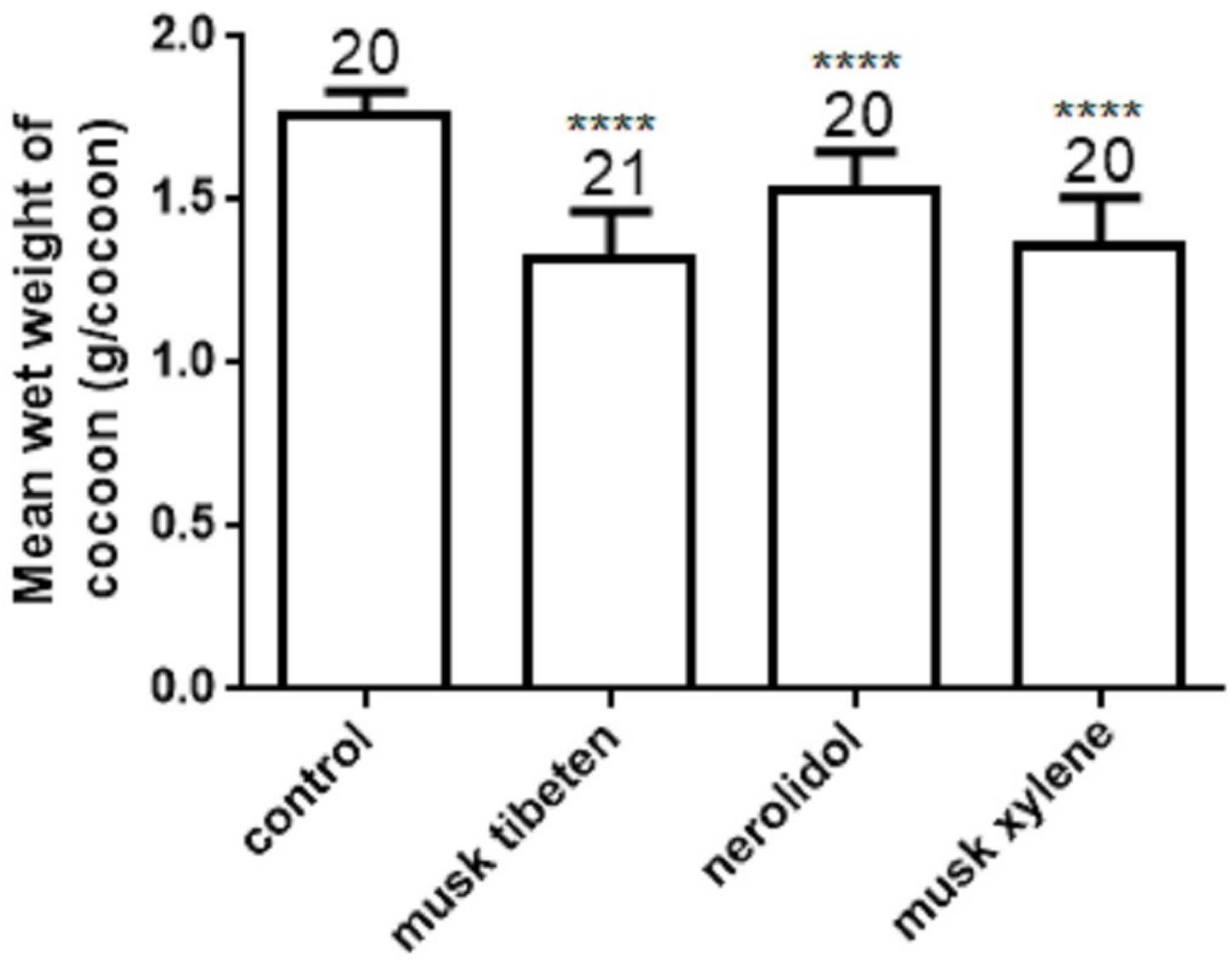


Figure 4

Effects of musk tibeten, nerolidol, and musk xylene on hemolymph JHs titer of newly molted fifth instar $B$. mori (day 0 ).

Values represent means $\pm \mathrm{SEM}, * * * * p<0.0001$

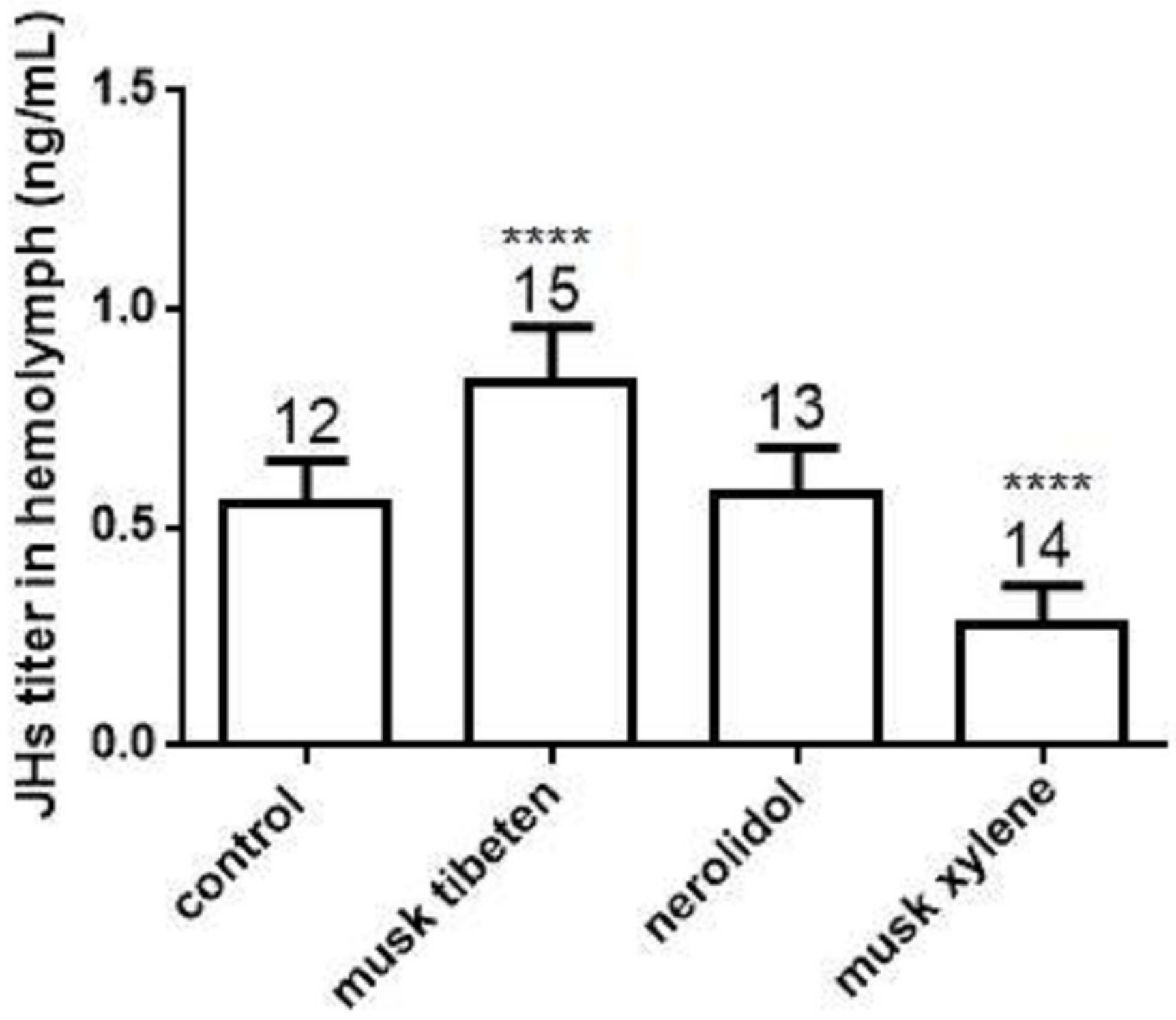


Figure 5

Effects of musk tibeten, nerolidol, and musk xylene on hemolymph 20-E titer of $B$. mori larvae on the fourth day of the fourth instar.

Values represent means \pm SEM, ${ }^{* * * *} p<0.0001$

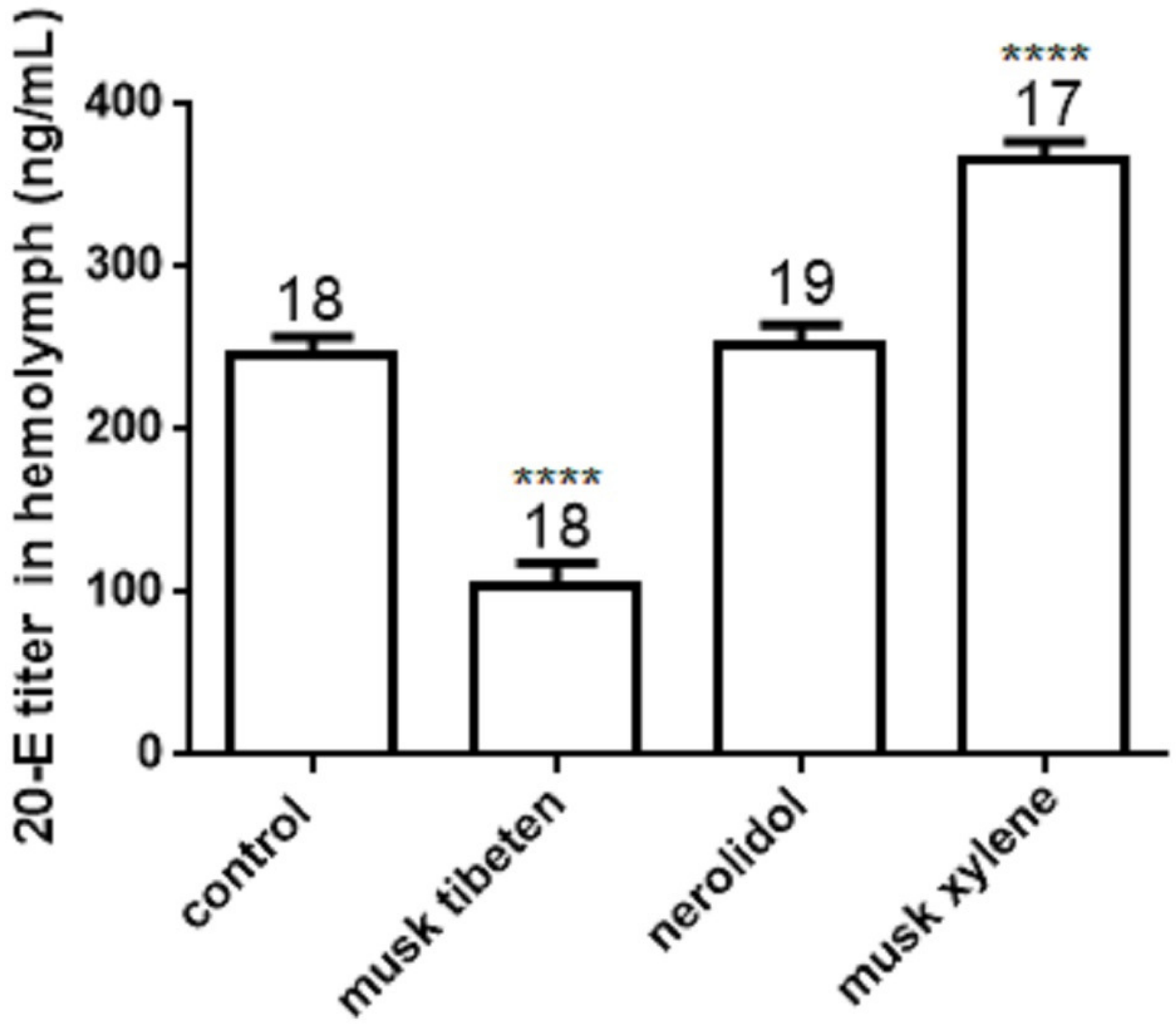

